\title{
STUDIO DELLA RADIOATTIVITA' DELLE LAVE DEL- L'ETNA COL METODO DELLE EMULSIONI NUCLEARI
}

\author{
L. Barbera - M. Curatolo - M. M. Indovina Addario - \\ M. Santangelo
}

1. - In questi ultimi tempi sono state eseguite diverse ricerche sulla radioattività dei materiali costituenti la crosta terrestre. Questo studio ha importanza in quanto può dare informazioni sui processi fisici e fisico-chimici, che interessano i problemi connessi con la formazione e con l'evoluzione della crosta terrestre (differenziazione dei magmi, calore radioattivo...). Tra i materiali solidi le lave vulcaniche presentano interesse particolare; due sono $\mathrm{i}$ problemi principali che si incontrano nello studio della radioattività delle lave: a) determinazione del rapporto delle concentrazioni di Uranio e di Torio: b) investigazione sull'esistenza o meno dello stato di equilibrio radioattivo dei componenti le due famiglie.

Recentemente sono state effettuate sulle lave del Vesuvio dclle misure $\left({ }^{1}\right)$, le quali confermano quanto era già stato affermato fin dal 1909 da Joly ( $\left.{ }^{2}\right)$, cioè che dette lave hanno un contenuto radioattivo dipendente dalla data d'eruzione. $E$ poiché è stato affermato che in lave di altri vulcani non è stata riscontrata fin'ora questa caratteristica, abbiamo ritenuto opportuno sottoporre ad esame quelle dell'Etna, per vedere se la loro attività dipendesse o no dalla data d'eruzione. Per eseguire queste misure abbiamo usato la tecnica delle emulsioni nucleari, tecnica già impiegata da molti autori per ricerche sulla radioattività delle rocce $\left({ }^{1}\right),\left({ }^{3}\right),\left({ }^{4}\right),\left({ }^{5}\right),\left({ }^{6}\right),\left({ }^{7}\right),\left({ }^{8}\right),\left({ }^{9}\right),\left({ }^{10}\right)$.

2. - Lastre Ilford $C_{\varrho}$, spesse $50 \mu$, sono state esposte per 51 giorni all'irraggiamento di sezioni levigate di lave, aventi uno spessore $>100_{u}$, cioè uno spessore tale da potersi ritenere infinito rispetto al range massimo delle particelle $\alpha$; tali lave appartengono a un'eruzione preistorica (lava I), a una del 1381 (lava II), a una del 1669 (lava III) e infine a una del 1928 (lava IV) ${ }^{11}$ ). Poiché l'attività delle lave in genere risulta molto debole, si è stimato necessario prolungare il tempo 
di esposizione; per questo abbiamo pensato fosse opportuno prendere alcune precauzioni per rendere minimo il fading, per perdere il minor numero possibile di particelle $\alpha$ e infine per diminuire il fondo. Non abliamo usato halsamo del Canada per attaccare le sezioni ai vetrini, dato che questo prodotto può sviluppare perossido d'idrogreno,

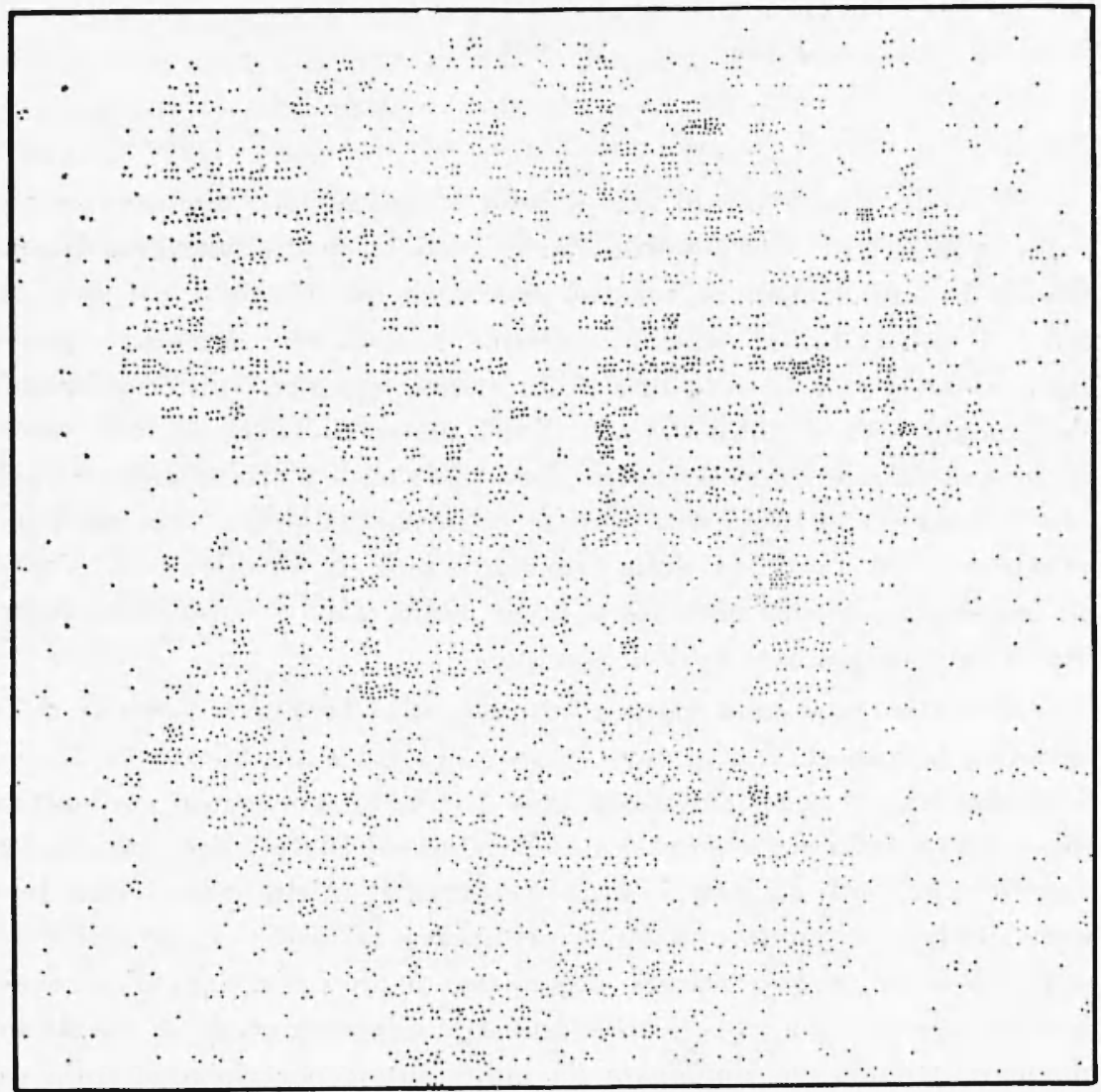

Fig. 1 - Mappa dell'illivita della lava I.

composto quest'ultimo che favorisce il fading $\left({ }^{12}\right)$ : ahbiamo attaccato perciò le sezioni con una colla di cellulosa vergine all'acetone.

Sempre per rendere minimo il fading abbiamo tenuto, durante tutto il periodo dell'esposizione, le lastre entro un essiccatore, contenente cloruro anidro di calcio, e in un ambiente mantenuto alla tem- 
peratura costante di $0{ }^{\circ} \mathrm{C}\left({ }^{-}\right)$. Allo scopo di ridurre gli effetti di fondo è stata ricoperta di paraffina la superficie della lastra non occupata dalla sezione.

Per mantenere le sezioni a contatto coll'emulsione fotografica e rendere minimo la strato d'aria tra sezione ed emulsione, abbiamo usato dei torchietti $\left({ }^{12}\right)$. Lo strato di gomma piuma è stato posto per rendere uniforme la pressione su tutta la superficie della lastra, in modo da evitare alterazioni.

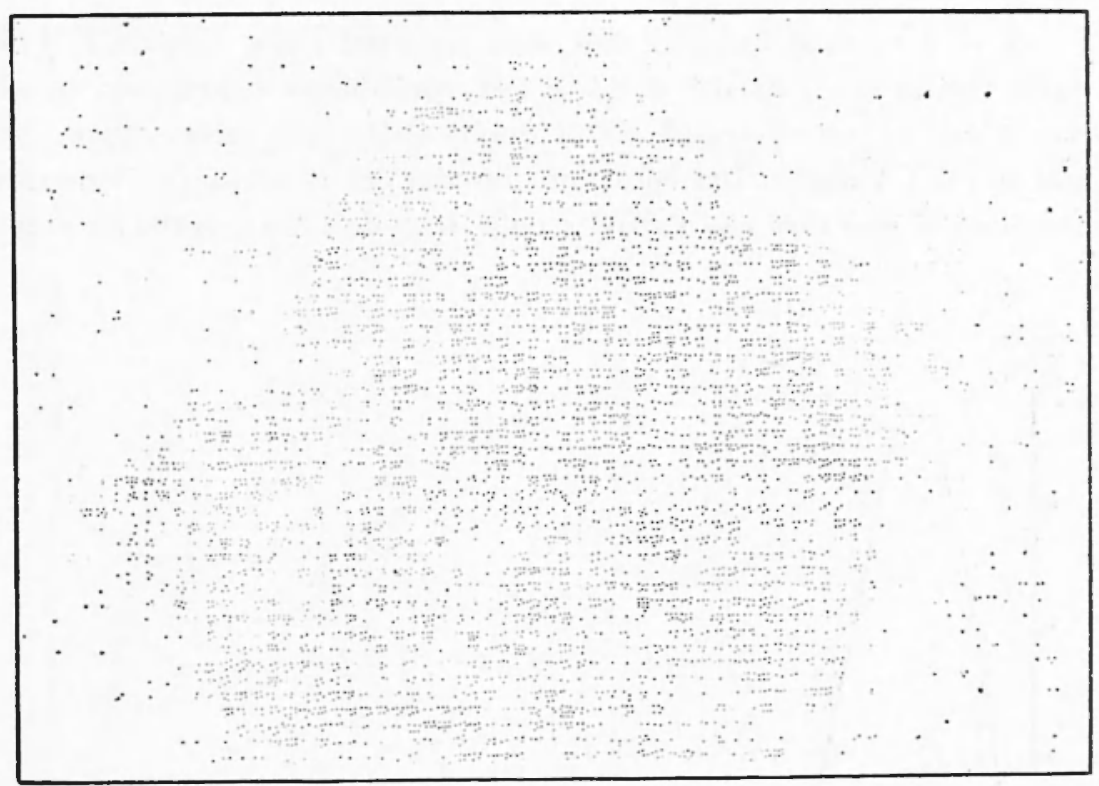

Fig. 2 - Mappa dell'attivití della lava III.

Nella costruzione dei torchietti abbiamo usato bachelite invece di un'altra sostanza, perché la bachelite ha un basso contenuto di impurità radioattive.

Le lastre sono state sviluppate, subito dopo l'esposizione, nel seguente modo:

1) sviluppo: $1 \mathrm{~g}$ Amidol, 8 g $\mathrm{Na}_{2} \mathrm{SO}_{3}, 340 \mathrm{~cm}^{3} \mathrm{H}_{2} \mathrm{O}:$ tempo 25 min; temperatura $16{ }^{\circ} \mathrm{C}$; nessuna agitazione;

2) lavaggio in acqua per $5 \mathrm{~min}$;

3) bagno di frenaggio: acido acetico al $2 \%$; tempo $15 \mathrm{~min}$.: temperatura $16{ }^{\circ} \mathrm{C}$ : 
4) fissaggio: iposolfito al $40 \%$; tempo circa 45 min.; temperatura $20^{\circ} \mathrm{C}$; nessuna agitazione;

5) lavaggio in acqua corrente per $15 \mathrm{~min}$.

L'esplorazione è stata eseguita con un ingrandimento di 460 dividendo la lastra in caselle di $330 \times 330 u^{2}$; di ogni lastra è stata eseguita una mappa (fig. 1 e fig. 2), riportando le particelle trovate in una casella di $330 \times 330 \mathrm{u}^{2}$ in un quadratino di $5 \times 5 \mathrm{~mm}^{2}$. Durante l'esplorazione sono state segnate solo le tracce di particelle $a$, aventi una proiezione orizzontale compresa tra $5 \mu$ e $50 \mu$.

3. - I risultati ottenuti sono stati riportati nella Tabella I, dalla quale risulta che l'attività delle sezioni considerate è piuttosto hassa; essa ̀̀ dell'ordine di grandezza di quella delle lave meno attive (più antiche) del Vesuvio. Dal confronto diretto dei risultati per i quattro campioni si può dire che l'attività delle lave dell'Etna, entro gli errori

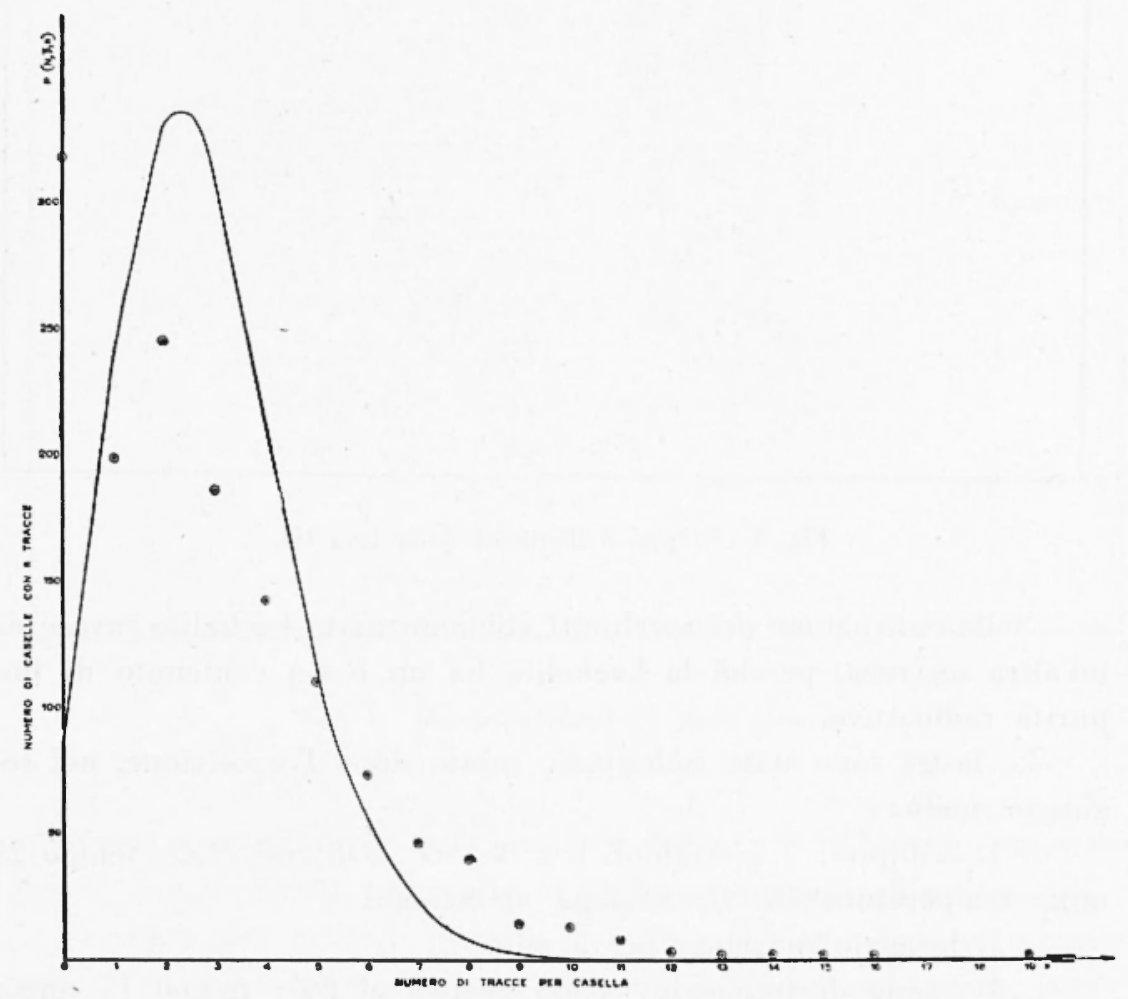

Fig. 3 - Lava I. 


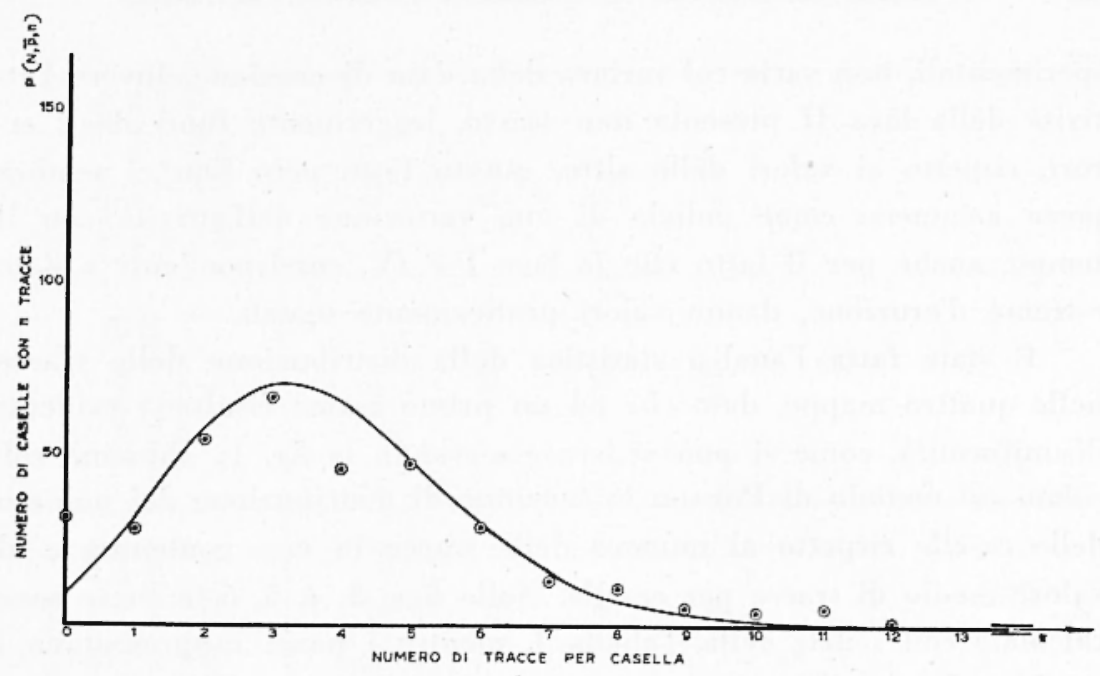

Fig. 4 - Lava II.

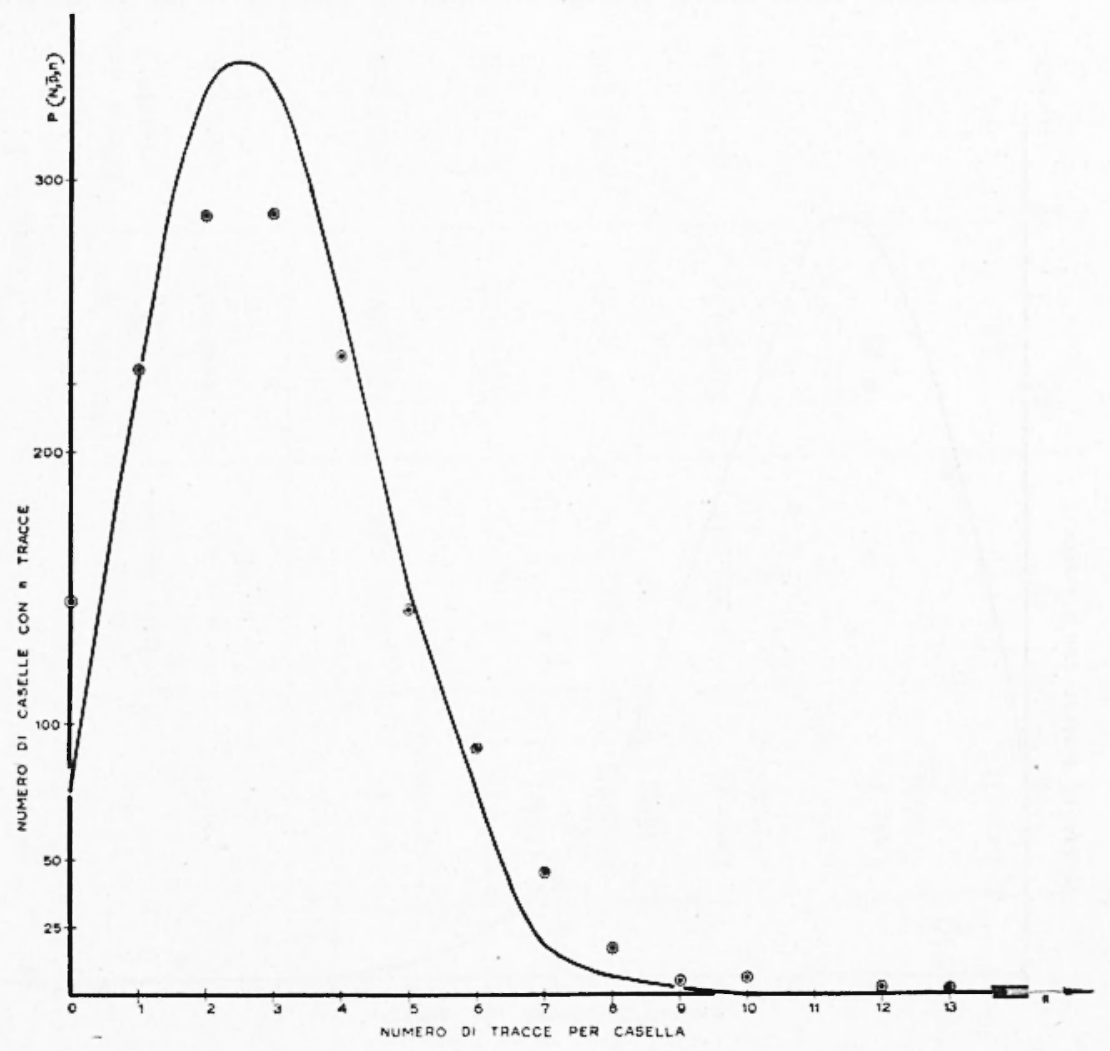

Fig. 5 - Lava III. 
sperimentali, non varia col variare della data di eruzione. Invero l'attività della lava II presenta uno scarto, leggermente fuori degli errori, rispetto ai valori delle altre, questo fatto però non ci sembra possa assumersi come indizio di una variazione dell'attività con il tempo, anche per il fatto che le lave I e IV, corrispondenti a date estreme d'eruzione, danno valori praticamente uguali.

E stata fatta l'analisi statistica della distribuzione delle tracce nelle quattro mappe, dato che ad un primo esame risultano evidenti disuniformità, come si può vedere osservando la fig. 1 ; abbiamo calcolato col metodo di Poisson la funzione di distribuzione del numero delle caselle rispetto al numero delle tracce in esse contenute e al valore medio di tracce per casella. Nelle figg. 3, 4, 5, 6 le curve sono calcolate con i dati della Tabella $I$, mentre i punti rappresentano i corrispondenti valori sperimentali.

Dall'esame delle curve risulta che per le lave II e III la distri-

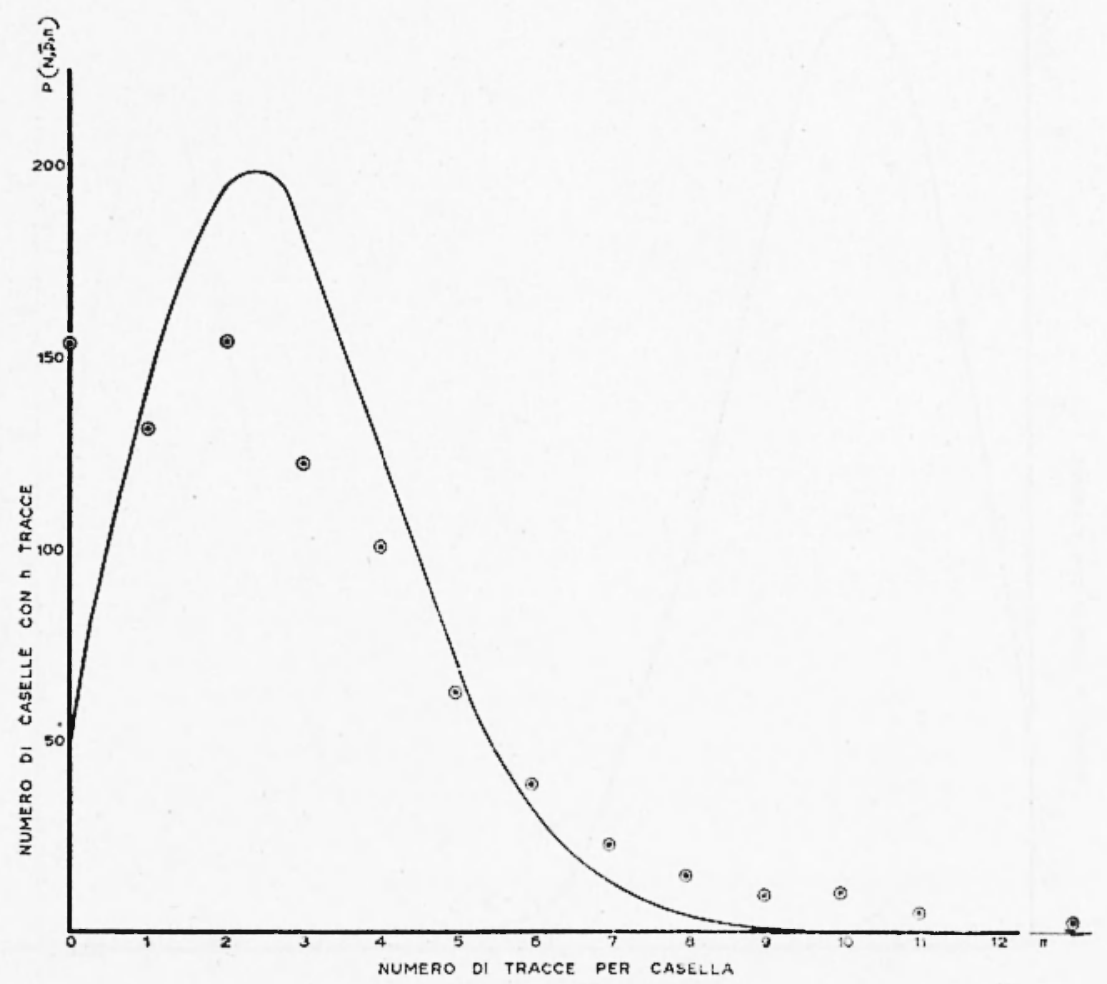

Fig. 6 - Lava IV. 


\begin{tabular}{|c|c|c|c|c|c|c|}
\hline 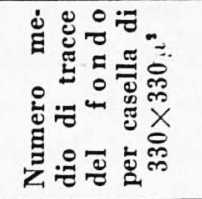 & $\ddot{c}$ & $\stackrel{12}{2}$ & $\stackrel{0}{\stackrel{0}{\circ}}$ & ศึ. & & \\
\hline 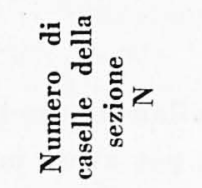 & 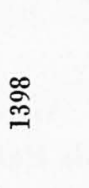 & लి & 冚 & $\stackrel{\ominus}{\infty}$ & & \\
\hline 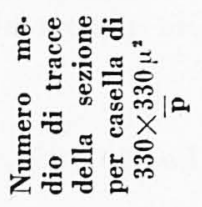 & $\underset{\sim}{\stackrel{i}{*}}$ & $\underset{\text { is }}{\stackrel{R}{n^{2}}}$ & बे & $\underset{\text { i }}{\infty}$ & & \\
\hline 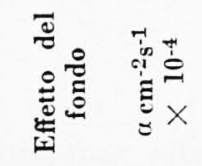 & \begin{tabular}{l} 
Õ \\
0 \\
+ \\
+1 \\
\multirow{2}{*}{} \\
0
\end{tabular} & 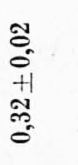 & $\begin{array}{l}\text { ò } \\
0 \\
+ \\
+1 \\
0 \\
0 \\
0\end{array}$ & 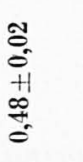 & & \\
\hline 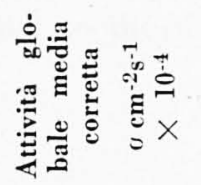 & 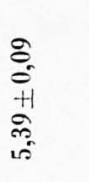 & 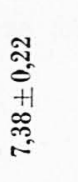 & $\begin{array}{l}\hat{E} \\
\text { के } \\
+1 \\
\text { हो }\end{array}$ & 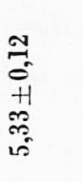 & ঞ & in \\
\hline \multirow[t]{3}{*}{ 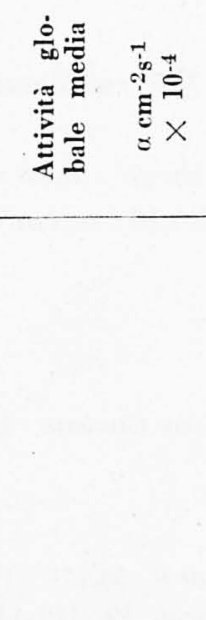 } & $\begin{array}{l}8 \\
0 \\
0 \\
+1 \\
0 \\
\text { is }\end{array}$ & $\begin{array}{l}\tilde{\sim} \\
\tilde{\sigma} \\
H \\
\tilde{n}\end{array}$ & 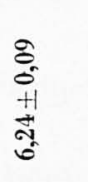 & 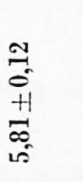 & & \\
\hline & ص离 & 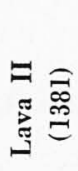 & 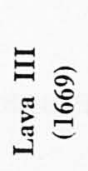 & 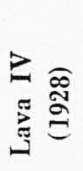 & 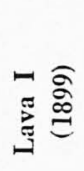 & $\stackrel{\Xi}{\stackrel{\Xi}{\Xi}}$ \\
\hline & & \multicolumn{2}{|c|}{ 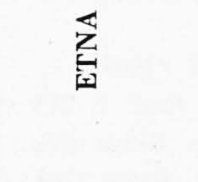 } & & \multicolumn{2}{|c|}{ 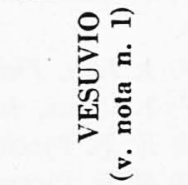 } \\
\hline
\end{tabular}


buzione sperimentale si adatta grosso modo a quella teorica di Poisson; infatti la mappa della lava III (fig. 2) si presenta con pochissime disuniformità. Invece la distribuzione sperimentale per le lave I e IV si allontana in modo più marcato da quella di Poisson: osservando la mappa della figura 1 , si vede che si hanno zone di addensamento di particelle a e zone di rarefazione.

4. - $\mathrm{Ci}$ è gradito ringraziare il prof. Angelo Bellanca, direttore dell'Istituto di Mineralogia dell'Università di Palermo, per avere messo a nostra disposizione parte dell'attrezzatura necessaria alla ricerca; ringraziamo pure la laureanda Maria Giardina per la preparazione delle sezioni sottili.

$$
\text { Palermo - Istituto di Fisica dell'Università -- Luglio } 1952 .
$$

\section{RIASSUNTO}

Si espongono $i$ primi risultati di uno studio sulla radioattività delle lave dell'Etna; si conclude che l'attività globale media di tali lave non varia con la data d'eruzione, essa ha un valore di $\sim 5-6.10^{-4}$ particelle a per cmi2 e per sec.

\section{SUMMARY}

The Authors give some preliminary results on the radionctivity of the "lave etnee".

They conclude that the total media activity of these "lave" do not change with the date of the eruption and it is $\sim 5-6.10^{-4} a_{/} \mathrm{cm}^{\circ} \mathrm{sec}$.

\section{BIBLIOGRAFIA}

(1) F. Bachelet, M. Ferretti Sforzini e F. Iprolito, Nuovo Cimento. 8, 851 (1951).

(2) J. Joly, Phil. Mag. 18, 577 (1909).

(3) I. Curie, Journ. Plyss. et Rad. 7, 313 (1946).

(1) E. E. Picciotto, Bull. Soc. Relge Géo. Paléont. el Hydrol. 58, 75 (1949).

(i) E. E. Picciotio, Bull. Soc. Belge Géo. Paléont. el Hydrol. 59, 170 (1950). 
(6) J. H. J. Poole e J. W. Bremmer, Nature. 163, 130 (1919).

(7) J. H. J. Poole e J. W. Brfameh, Nature. 161, 881 (1948).

(8) I. H. Fond, Nature. 167, 273 (1951).

(9) S. Milone, Tamburino e A. Stella, Nuovo Cimento. 9, 253 (1952).

(10) H. V. Blttlar e F. G. Houtermans, Geochim. et Cosmochim. Actu, 2, 13 (1951).

(11) Ringraziamo il prof. G. Ponte per averci gentilmente fornito i suddetti ampioni di lava.

(12) H. Yatoda, Radioactive Measurementes with Nuclear Emulsions. (New ork, 1949). 